\title{
Two-photon probe of the Jaynes-Cummings model and controlled symmetry breaking in circuit QED
}

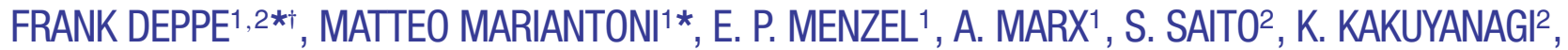
H. TANAKA ${ }^{2}$, T. MENO ${ }^{3}$, K. SEMBA ${ }^{2}$, H. TAKAYANAGI ${ }^{4,5}$, E. SOLANO ${ }^{6,7}$ AND R. GROSS ${ }^{1}$

\author{
${ }^{1}$ Walther-Meißner-Institut, Bayerische Akademie der Wissenschaften, Walther-Meißner-Str. 8, D-85748 Garching, Germany \\ ${ }^{2}$ NTT Basic Research Laboratories, NTT Corporation, Kanagawa, 243-0198, Japan \\ ${ }^{3}$ NTT Advanced Technology, NTT Corporation, Kanagawa, 243-0198, Japan \\ ${ }^{4}$ Tokyo University of Science, 1-3 Kagurazaka, Shinjuku, Tokyo, 162-8601, Japan \\ ${ }^{5}$ International Center for Materials Nanoarchitectronics, NIMS, Tsukuba 305-0003, Japan \\ ${ }^{6}$ Physics Department, ASC and CeNS, Ludwig-Maximilians-Universität, Theresienstr. 37, 80333 München, Germany \\ ${ }^{7}$ Departamento de Química Física, Universidad del País Vasco - Euskal Herriko Unibertsitatea, Apdo. 644, 48080 Bilbao, Spain \\ *These authors contributed equally to this work \\ †e-mail: frank.deppe@wmi.badw-muenchen.de
}

Superconducting qubits ${ }^{1,2}$ behave as artificial two-level atoms and are used to investigate fundamental quantum phenomena. In this context, the study of multiphoton excitations ${ }^{3-7}$ occupies an important role. Moreover, coupling superconducting qubits to onchip microwave resonators has given rise to the field of circuit quantum electrodynamics ${ }^{8-15}$ (QED). In contrast to quantum-optical cavity QED (refs 16-19), circuit QED offers the tunability inherent to solid-state circuits. Here, we report on the observation of key signatures of a two-photon-driven Jaynes-Cummings model, which unveils the upconversion dynamics of a superconducting flux qubit ${ }^{20}$ coupled to an on-chip resonator. Our experiment and theoretical analysis show clear evidence for the coexistence of one- and two-photon-driven level anticrossings of the qubit-resonator system. This results from the controlled symmetry breaking of the system hamiltonian, causing parity to become a not-well-defined property $^{21}$. Our study provides fundamental insight into the interplay of multiphoton processes and symmetries in a qubit-resonator system.

In cavity quantum electrodynamics (QED), a two-level atom interacts with the quantized modes of an optical or microwave cavity. The information on the coupled system is encoded both in the atom and in the cavity states. The latter can be accessed spectroscopically by measuring the transmission properties of the cavity $^{16}$, whereas the former can be read out by suitable detectors $^{18,19}$. In circuit QED, the solid-state counterpart of cavity QED, the first category of experiments was implemented by measuring the microwave radiation emitted by a resonator (acting as a cavity) strongly coupled to a charge qubit ${ }^{8}$. In a dual experiment, the state of a flux qubit was detected with a d.c. superconducting quantum interference device (SQUID) and vacuum Rabi oscillations were observed ${ }^{10}$. More recently, both approaches have been exploited to extend the toolbox of quantum optics on a chip ${ }^{11-15,22}$. Whereas all of these experiments use one-photon driving of the coupled qubit-resonator system, multiphoton studies are available only for sideband transitions ${ }^{15}$ or bare qubits ${ }^{3-7}$. The experiments discussed here explore, to our knowledge for the first time, the physics of the two-photon-driven Jaynes-Cummings dynamics in circuit QED. In this context, we show that the dispersive interaction between the qubit and the two-photon driving enables real level transitions. The nature of our experiment can be understood as an upconversion mechanism, which transforms the two-photon coherent driving into single photons of the Jaynes-Cummings dynamics. This process requires energy conservation and a not-well-defined parity ${ }^{21}$ of the interaction hamiltonian owing to the symmetry breaking of the qubit potential. Our experimental findings reveal that such symmetry breaking can be obtained either in a controlled way by choosing a suitable qubit operation point or by the presence of spurious fluctuators ${ }^{23}$.

The main elements of our set-up, shown in Fig. 1a,b, are a three-Josephson-junction flux qubit, a resonator made of an inductor $L$ and a capacitor $C$ ( $L C$ resonator), a d.c. SQUID and a microwave antenna ${ }^{24,25}$. The qubit is operated near the optimal flux bias $\Phi_{\mathrm{x}}=1.5 \Phi_{0}$ and can be described with the hamiltonian $\hat{H}_{\mathrm{q}}=\left(\epsilon \hat{\sigma}_{z}+\Delta \hat{\sigma}_{x}\right) / 2$, where $\hat{\sigma}_{x}$ and $\hat{\sigma}_{z}$ are Pauli operators. From low-level microwave spectroscopy, we estimate a qubit gap $\Delta / h=3.89 \mathrm{GHz}$. By changing $\Phi_{\mathrm{x}}$, the quantity $\epsilon \equiv 2 I_{\mathrm{p}}\left(\Phi_{\mathrm{x}}-1.5 \Phi_{0}\right)$ and, in turn, the level splitting $\hbar \omega_{\mathrm{q}} \equiv \sqrt{\epsilon^{2}+\Delta^{2}}$ can be controlled. Here, $\pm I_{\mathrm{p}}$ are the clockwise and anticlockwise circulating persistent currents associated with the eigenstates $| \pm\rangle$ of $\epsilon \hat{\sigma}_{z}$. Far from the optimal point, $| \pm\rangle$ correspond to the eigenstates $|\mathrm{g}\rangle$ and $|\mathrm{e}\rangle$ of $\hat{H}_{\mathrm{q}}$. The qubit is inductively coupled to a lumped-element $L C$ resonator ${ }^{10}$, which can be represented by a quantum harmonic oscillator (see the Methods section), $\hat{H}_{\mathrm{r}}=\hbar \omega_{\mathrm{r}}\left(\hat{a}^{\dagger} \hat{a}+1 / 2\right)$, with photon number states $|0\rangle,|1\rangle$, $|2\rangle, \ldots$ and boson creation and annihilation operators $\hat{a}^{\dagger}$ and $\hat{a}$ respectively. This resonator is designed such that its fundamental 

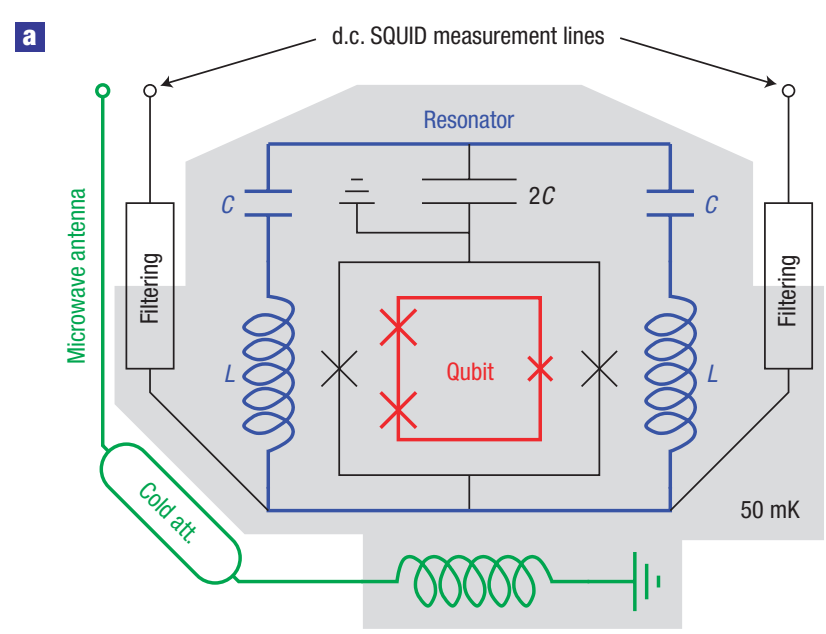

b

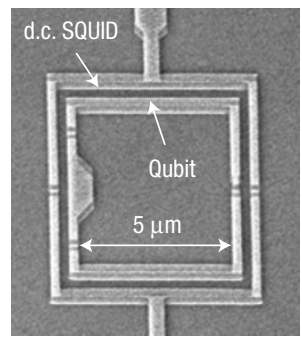

G

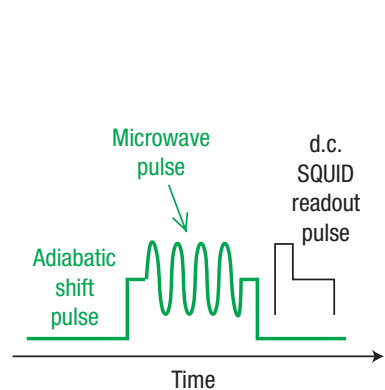

d
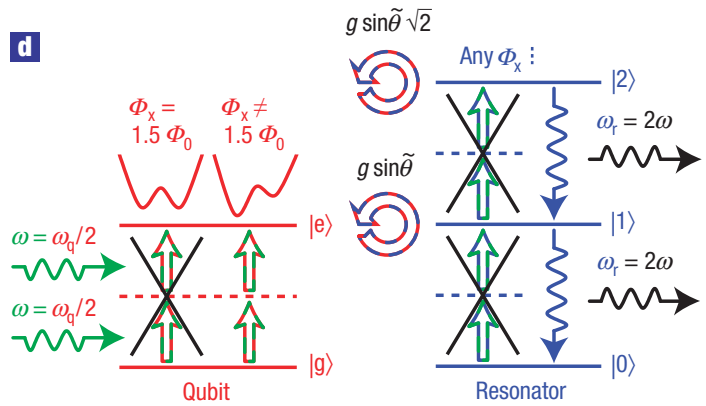

Figure 1 Experimental architecture and theoretical model. a, The flux qubit (red, junctions marked by crosses) is inductively coupled to the readout d.c. SQUID (black rectangle), which is shunted by an $L C$ circuit acting as a quantized resonator (blue) $)^{10}$. All elements within the shaded area are at a temperature $T \simeq 50 \mathrm{mK}$. Microwave signals and flux-shift pulses are applied via an onchip antenna (green). The signal-to-noise ratio is improved by cold attenuators. $\mathbf{b}$, Scanning electron micrograph of flux qubit and readout d.c. SQUID. c, Pulse scheme for qubit microwave spectroscopy with the adiabatic shift pulse method. First, the qubit is biased at a suitable readout point $\left(\Phi_{\mathrm{x}} \neq 1.5 \Phi_{0}\right)$ using a superconducting coil and initialized in the ground state $|\mathrm{g}\rangle$. Then, the qubit is shifted to the operation point with a rectangular adiabatic shift pulse (green). There, it is irradiated with a $100 \mathrm{~ns}$ microwave pulse (green). Finally, the qubit state is detected by measuring $l_{p} \hat{\sigma}_{z}$ at the readout point applying a pulse to the d.c. SQUID measurement lines. Averaging yields the probability $P_{\mathrm{e}}$ to find the qubit in the excited state $|\mathrm{e}\rangle$. Our protocol enables qubit-state detection also at the optimal point ${ }^{24}$ despite a vanishing mean value $I_{p}\left\langle\hat{\sigma}_{z}\right\rangle$. d, Upconversion dynamics describing the physics governing our experiments, see equation (1). The qubit (red) level splitting is $\hbar \omega_{\mathrm{q}}$ and the resonator (blue) frequency is $\omega_{\mathrm{r}} / 2 \pi$. In the relevant case of two-photon driving with frequency $\omega / 2 \pi$ (green), the system predominantly decays via the resonator. The qubit-resonator coupling strength is $g \sin \tilde{\theta}=g \Delta / \omega_{\mathrm{r}} \simeq 0.63 g$. For $\Phi_{\mathrm{x}} \neq 1.5 \Phi_{0}$, the mirror symmetry of the qubit potential (red double well; $x$ axis: phase variable $\hat{\varphi}_{\mathrm{m}}$ ) is broken enabling two-photon transitions.

frequency, $\omega_{\mathrm{r}} / 2 \pi=6.16 \mathrm{GHz}$, is largely detuned from $\Delta / h$. The qubit-resonator interaction hamiltonian is $\hat{H}_{\mathrm{q}, \mathrm{r}}=\hbar g \hat{\sigma}_{z}\left(\hat{a}^{\dagger}+\hat{a}\right)$, where $g=2 \pi \times 115 \mathrm{MHz}$ is the coupling strength. The $L C$ circuit also constitutes a crucial part of the electromagnetic environment of the readout d.c. SQUID. In this way, the flux signal associated with the qubit states $| \pm\rangle$ can be detected while maintaining reasonable coherence times and measurement fidelity ${ }^{24,25}$.

To probe the properties of our system, we carry out qubit microwave spectroscopy using an adiabatic shift pulse technique ${ }^{24,25}$ (Fig. 1c). The main results are shown in Fig. 2a,b. First, there is a flux-independent feature at approximately $6 \mathrm{GHz}$ due to the resonator. Second, we observe two hyperbolas with minima near $4 \mathrm{GHz} \simeq \Delta / h$ and $2 \mathrm{GHz} \simeq \Delta / 2 h$, one with a broad and the other with a narrow linewidth. They correspond to the one-photon $\left(\omega=\omega_{\mathrm{q}}\right)$ and two-photon $\left(2 \omega=\omega_{\mathrm{q}}\right)$ resonance condition between the qubit and the external microwave field. In addition, the signatures of two-photon-driven blue-sideband transitions are partially visible. One can be attributed to the resonator, $|g, 0\rangle \rightarrow|e, 1\rangle$, and the other to a spurious fluctuator ${ }^{23}$. We assume that the latter is represented by the flux-independent hamiltonian $\hat{H}_{\mathrm{f}}=\left(\epsilon^{\star} \hat{\sigma}_{z}^{\star}+\Delta^{\star} \hat{\sigma}_{x}^{\star}\right) / 2$ and coupled to the qubit via $\hat{H}_{\mathrm{q}, \mathrm{f}}=\hbar g^{\star} \hat{\sigma}_{z} \hat{\sigma}_{z}^{\star}$, where $\hat{\sigma}_{x}^{\star}$ and $\hat{\sigma}_{z}^{\star}$ are Pauli operators. Exploiting the different response of the system in the anticrossing region under one- and two-photon driving, as explained in Fig. 2a, the centre frequencies of the spectroscopic peaks can be accurately fitted to the undriven hamiltonian $\hat{H}_{\mathrm{u}}=\hat{H}_{\mathrm{q}}+\hat{H}_{\mathrm{r}}+\hat{H}_{\mathrm{f}}+\hat{H}_{\mathrm{q}, \mathrm{r}}+\hat{H}_{\mathrm{q}, \mathrm{f}}$. Setting $\epsilon^{\star}=0$ (see the Methods section), we obtain $g / 2 \pi=115 \mathrm{MHz}$, $\langle\hat{N}\rangle \simeq 10, I_{\mathrm{p}}=367 \mathrm{nA}, \omega_{\mathrm{f}} / 2 \pi \equiv \sqrt{\epsilon^{\star 2}+\Delta^{\star^{2}}} / h=3.94 \mathrm{GHz}$ and $g^{\star} \sin \theta^{\star}=37 \mathrm{MHz}$, where $\sin \theta^{\star} \equiv \Delta^{\star} / \hbar \omega_{\mathrm{f}}$.

Further insight into our experimental results can be gained by numerical spectroscopy simulations based on the driven hamiltonian $\hat{H}_{\mathrm{d}}=\hat{H}_{\mathrm{u}}+\hat{H}_{\mathrm{m}, \mathrm{q}}+\hat{H}_{\mathrm{m}, \mathrm{r}}+\hat{H}_{\mathrm{m}, \mathrm{f}}$. Here, $\hat{H}_{\mathrm{m}, \mathrm{q}}=(\Omega / 2) \hat{\sigma}_{z} \cos \omega t, \hat{H}_{\mathrm{m}, \mathrm{r}}=\eta\left(\hat{a}^{\dagger}+\hat{a}\right) \cos \omega t$ and $\hat{H}_{\mathrm{m}, \mathrm{f}}=\left(\Omega^{\star} / 2\right) \hat{\sigma}_{z}^{\star} \cos \omega t$ represent the driving of the qubit, resonator and fluctuator respectively. We approximate the steady state with the time average of the probability $P_{\mathrm{e}}$ to find the qubit in $|e\rangle$ (time-trace-averaging method). Inspecting Fig. 2c, we find that for the driving strengths $\Omega / h=244 \mathrm{MHz}, \eta / h=655 \mathrm{MHz}$ and $\Omega^{\star}=0$, our simulations match well all of the experimental features discussed above. Using $\eta$ and the relation $\langle\hat{N}\rangle=(\eta / \kappa)^{2}$ 

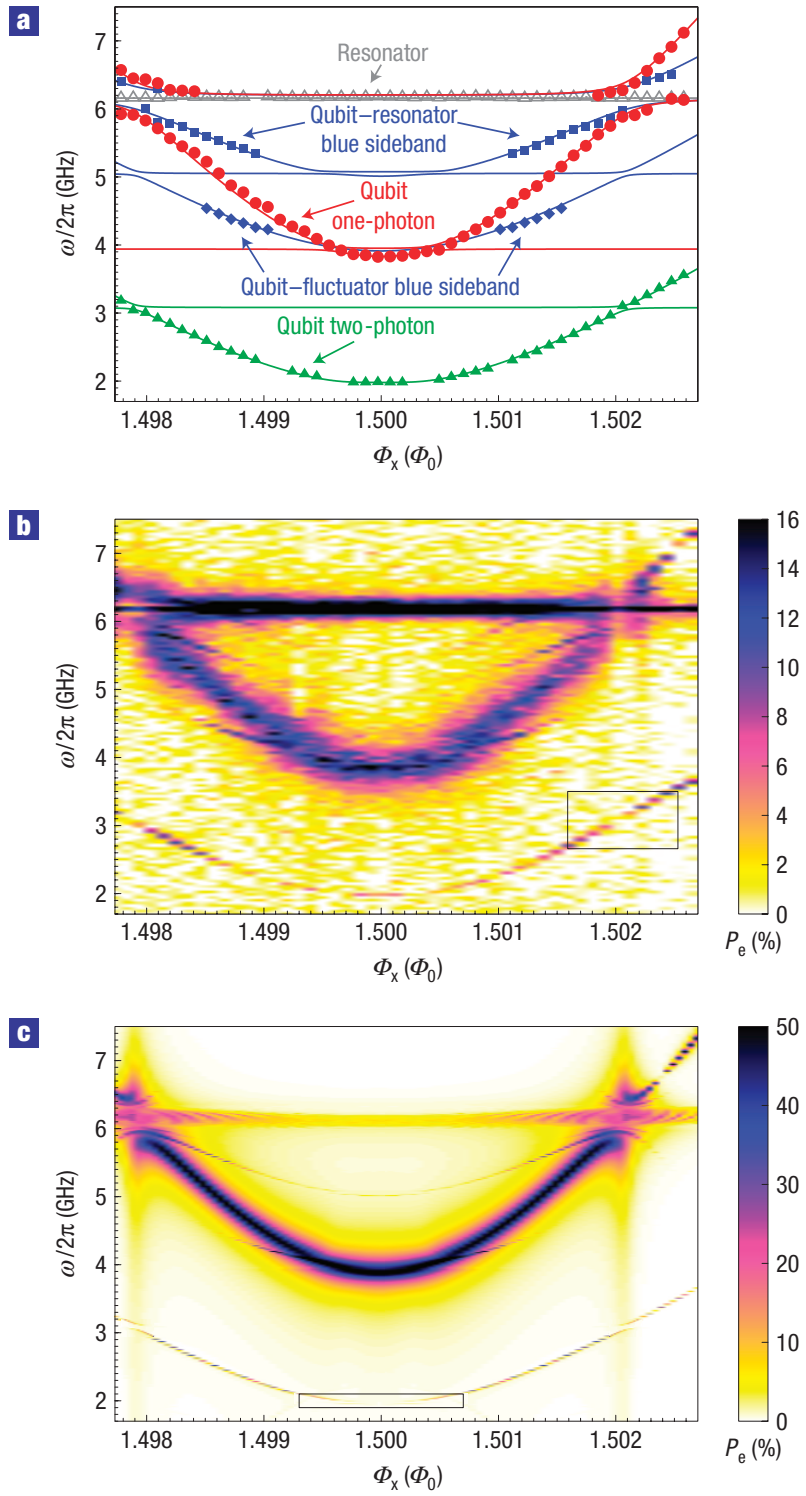

Figure 2 Qubit microwave spectroscopy: data and simulations. a, Centre frequency of the measured absorption peaks (symbols) plotted versus the flux bias. The lines are fits of the undriven hamiltonian $\hat{H}_{u}$ to the data. The presence of a large $\left(\omega \approx \omega_{\mathrm{r}}\right)$ and small $\left(2 \omega \approx \omega_{\mathrm{r}}\right)$ anticrossing constitutes direct evidence that two-photon spectroscopy selectively drives the qubit (but not the resonator), thereby probing the vacuum Rabi coupling $g$. On the contrary, the one-photon driving populates the cavity resulting in an enhanced coupling $g\langle\hat{M}\rangle^{1 / 2}$. b. Measured probability $P_{\mathrm{e}}$ to find the qubit in the excited state plotted versus flux bias and driving frequency (black rectangle: area shown in Fig. 3a). c, Simulated probability $P_{\mathrm{e}}$ obtained with the time-trace-averaging method for the driven hamiltonian $\hat{H}_{\mathrm{d}}$ (black rectangle: area shown in Fig. 4). Taking $P_{\mathrm{e}}$ as the average over a full $100 \mathrm{~ns}$ time trace consisting of 10,000 time points gives excellent agreement with the experimental data of $\mathbf{b}$. No terms describing dissipation are included; the linewidth of the peaks is caused by power broadening. Simulation parameters are derived from the fit in $\mathbf{a}$. The used inductance values are based on numerical estimates.

for the steady-state mean number of photons of a driven dissipative cavity, we estimate a cavity decay rate $\kappa \simeq 210 \mathrm{MHz}$. This result is of the same order as $\kappa \simeq 400 \mathrm{MHz}$ estimated directly from the experimental linewidth of the resonator peak. The large $\kappa$ is due a

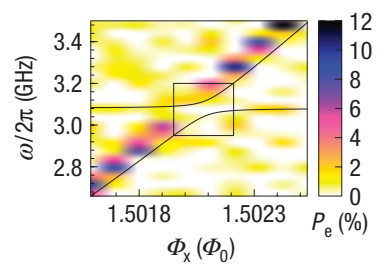

c

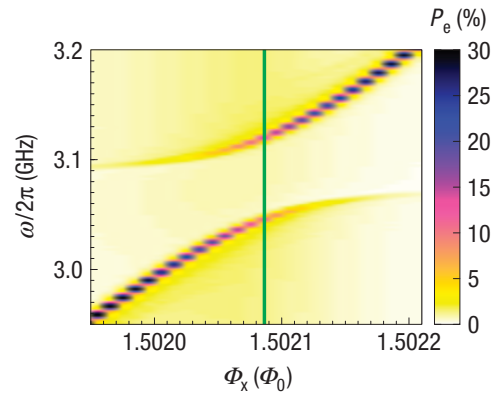

e

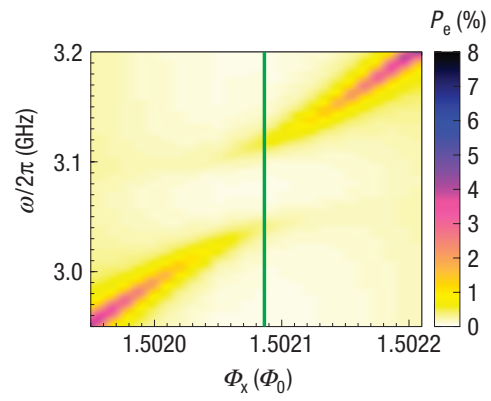

b

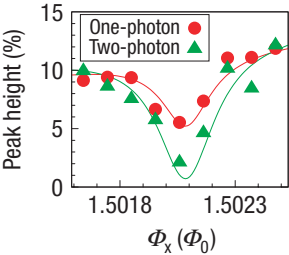

d

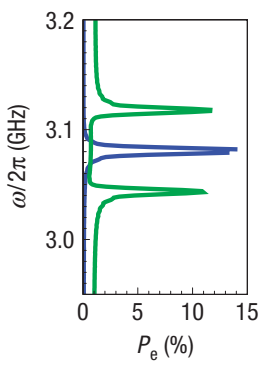

$\mathbf{f}$

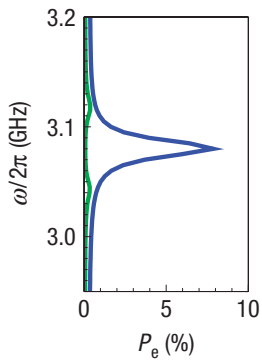

Figure 3 Qubit microwave spectroscopy close to the qubit-resonator anticrossing under two-photon driving: data and simulations. a, Measured probability $P_{\mathrm{e}}$ to find the qubit in $|\mathrm{e}\rangle$ plotted versus flux bias and driving frequency (black rectangle: area of simulations in c and e; solid lines: fit to $\hat{H}_{u}$ ). b. Maximum height of the spectroscopy peaks under one-photon and two-photon driving plotted versus the flux bias (solid lines: guides to the eye). c, Simulated probability $P_{\mathrm{e}}$ (time-trace-averaging method, no dissipation, parameters as in Fig. 2c), revealing an anticrossing signature. d, Green curve: split-peak profile of $P_{\mathrm{e}}$ along the vertical line in $\mathbf{c}$. Blue curve: single-peak result obtained for the same flux bias and $g=0$. e, Simulated probability $P_{\mathrm{e}}$ using the Lindblad formalism ${ }^{22}$ neglecting the spurious fluctuator (dissipation: qubit relaxation rate $\gamma_{1}=3.3 \mathrm{MHz}$, qubit dephasing rate $\gamma_{\varphi}=67 \mathrm{MHz}$, resonator quality factor

$Q \equiv \omega_{\mathrm{r}} / \kappa=2 \pi \times 6.16 \mathrm{GHz} / 400 \mathrm{MHz} \simeq 100$ ). When qubit and resonator become degenerate, the spectroscopy signal fades away. $\mathbf{f}$, Green curve: split-peak profile of $P_{\mathrm{e}}$ along the vertical line in e. Blue line: single-peak result obtained for the same flux bias and $g=0$. Differently from the non-dissipative case (c and $\mathbf{d}$ ), the split-peak amplitudes are reduced by a factor of 10 compared with the single peak. This demonstrates that the vanishing two-photon spectroscopy signal observed in the experimental data (see $\mathbf{a}, \mathbf{b}$ and $\mathbf{e}$ ) is not caused by qubit decoherence.

to the galvanic connection of the resonator to the d.c. SQUID measurement lines (Fig. 1a).

To elucidate the two-photon driving physics of the qubit-resonator system, we consider the spectroscopy data near the corresponding anticrossing shown in Fig. 3a. For $2 \omega=\omega_{\mathrm{q}}=\omega_{\mathrm{r}}$, the split peaks cannot be observed directly because the spectroscopy signal is decreased below the noise floor $\delta P_{\mathrm{e}} \simeq 1-2 \%$. This results from the fact that the resonator cannot absorb a two-photon driving and its excitation energy is rapidly lost to the environment $(\kappa>g / 2 \pi)$. In contrast, for the one-photon case 

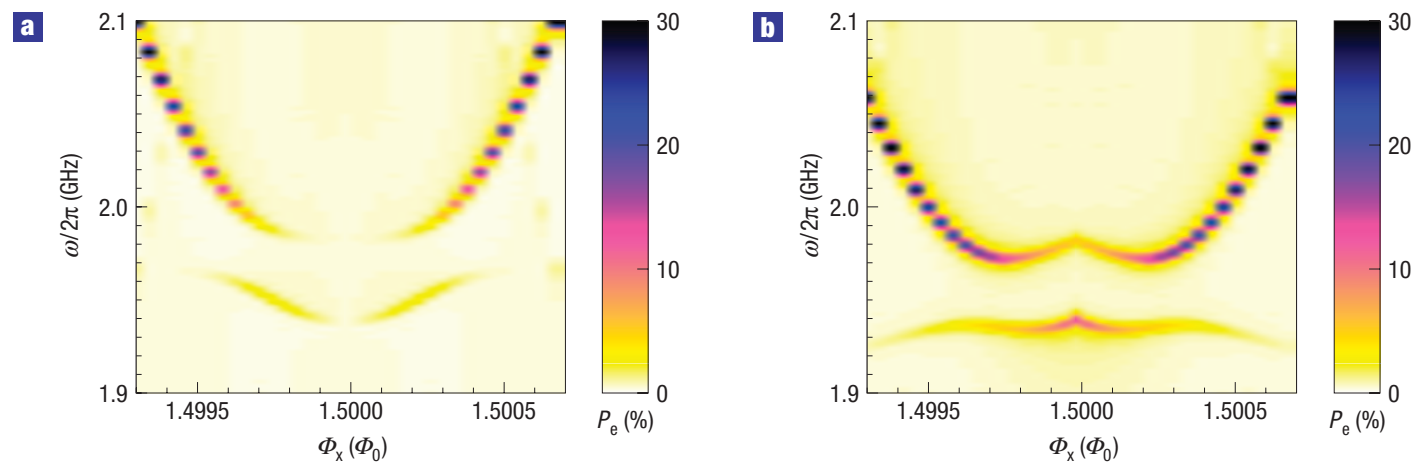

Figure 4 Two-photon spectroscopy simulations close to the optimal point using the time-trace-averaging method. a, Probability $P_{\mathrm{e}}$ to find the qubit in $|\mathrm{e}\rangle$ plotted versus driving frequency and flux bias (parameters as in Fig. 2c; in particular, the fluctuator parameters are $\epsilon^{\star}=0 \leftrightarrow \sin \theta^{\star}=1$ and $\Omega^{\star}=0$ ). The spectroscopy signal vanishes completely at the optimal point, $\Phi_{\mathrm{x}}=1.5 \Phi_{0}$, because of the specific selection rules associated with the symmetry properties of the hamiltonian ${ }^{21}$. $\mathbf{b}$, Same as in $\mathbf{a}$, but for $\sin \theta^{\star}=0.3$ and $\Omega^{\star}=280 \mathrm{MHz}$. Here, the coexistence of the flux-independent first-order $\hat{\sigma}_{x}^{\star}$ and $\hat{\sigma}_{z}^{\star}$ terms of the fluctuator gives rise to a non-vanishing second-order $\hat{\sigma}_{x}^{\star}$ term even at the qubit optimal point. The presence of the fluctuator breaks the symmetry of the total system at the optimal point and parity becomes not well defined. Consequently, the spectroscopy signal is partially revived. In reality, an ensemble of fluctuators with some distribution of frequencies and coupling strengths rather than a single fluctuator is expected to contribute to the symmetry breaking. Furthermore, when the experimental resolution is limited, a single peak will be detected instead of the detailed structure of $\mathbf{b}$. This is the case in our measurements (Fig. 2b).

$\left(\omega=\omega_{\mathrm{q}}=\omega_{\mathrm{r}}\right)$, there is a driving-induced steady-state population of $\langle\hat{N}\rangle \simeq 10$ photons in the cavity. Accordingly, the one-photon peak height shows a reduction by a factor of approximately two, whereas the two-photon peak almost vanishes, see Fig. 3b. To support this interpretation, we compare the simulation results from the time-trace-averaging method to those obtained with the standard Lindblad dissipative-bath approach (Fig. 3c-f). In the latter case, the role of qubit decoherence and resonator decay can be studied explicitly solving a master equation ${ }^{22}$. The simulation results of Fig. $3 \mathrm{c}-\mathrm{f}$ prove that the two-photon peak indeed vanishes because of the rapid resonator decay, but not because of qubit decoherence. Altogether, our experimental data and numerical simulations constitute clear evidence for the presence of a qubit-resonator anticrossing under two-photon driving.

The second-order effective hamiltonian under two-photon driving can be derived using a Dyson-series approach (see the Methods section). Starting from the first-order-driven hamiltonian $\hat{H}_{\mathrm{d}}$ and neglecting the cavity driving and the fluctuator because of large-detuning conditions, we obtain

$$
\begin{aligned}
\hat{H}^{(2)}= & \frac{\hbar \omega_{\mathrm{q}}}{2} \hat{\sigma}_{z}+\frac{\Omega^{2}}{4 \Delta} \sin ^{2} \theta \cos \theta\left(\hat{\sigma}_{+} \mathrm{e}^{-i 2 \omega t}+\hat{\sigma}_{-} \mathrm{e}^{+i 2 \omega t}\right) \\
& -\hbar g \sin \theta\left(\hat{\sigma}_{+} \hat{a}+\hat{\sigma}_{-} \hat{a}^{\dagger}\right)+\hbar \omega_{\mathrm{r}}\left(\hat{a}^{\dagger} \hat{a}+\frac{1}{2}\right),
\end{aligned}
$$

where $\hat{\sigma}_{+}$and $\hat{\sigma}_{-}$are the qubit raising and lowering operators respectively, $\sin \theta \equiv \Delta / \omega_{\mathrm{q}}$ and $\cos \theta \equiv \epsilon / \omega_{\mathrm{q}}$. The upconversion dynamics shown in Fig. $1 \mathrm{~d}$ is clearly described by equation (1). The first two terms represent the qubit and its coherent two-photon driving with angular frequency $\omega$. The last two terms show the population transfer via the Jaynes-Cummings interaction to the resonator. The Jaynes-Cummings interaction in this form is valid only near the anticrossings $\left(\theta \approx \tilde{\theta}, \tilde{\theta} \equiv\left(\Delta / \omega_{\mathrm{r}}\right) \simeq 0.63\right.$; see the Methods section). As discussed before, the resonator will then decay emitting radiation of angular frequency $2 \omega$.

The model outlined above enables us to unveil the symmetry properties of our system. Even though the two-photon coherent driving is largely detuned, $\omega_{\mathrm{q}} / 2=\omega \gg(\Omega / 2) \sin \theta$, a not-well-defined symmetry of the qubit potential permits level transitions away from the optimal point. Because of energy conservation, that is, frequency matching, these transitions are real and can be used to probe the qubit-resonator anticrossing. The effective two-photon qubit driving strength, $\left(\Omega^{2} \sin ^{2} \theta / 4 \Delta\right) \cos \theta$, has the typical structure of a second-order dispersive interaction with the extra factor $\cos \theta$. The latter causes this coupling to disappear at the optimal point. There, the qubit potential is symmetric and the parity of the interaction operator is well defined. Consequently, selection rules similar to those governing electric dipole transitions hold ${ }^{21}$. This is best understood in our analytical two-level model, where the first-order hamiltonian for the driven diagonalized qubit becomes $\hat{H}_{\mathrm{OP}}^{(1)}=(\Delta / 2) \hat{\sigma}_{z}+(\Omega / 4) \hat{\sigma}_{x}\left(\mathrm{e}^{+i \omega t}+\mathrm{e}^{-i \omega t}\right)$ at the optimal point. In this case, one-photon transitions are allowed because the driving couples to the qubit via the odd-parity operator $\hat{\sigma}_{x}$. In contrast, the two-photon driving effectively couples via the second-order hamiltonian $\hat{H}_{\mathrm{OP}}^{(2)}=(\Delta / 2) \hat{\sigma}_{z}+\left(\Omega^{2} / 16 \Delta\right) \hat{\sigma}_{z}\left(\mathrm{e}^{+i \omega t}+\mathrm{e}^{-i \omega t}\right)^{2}$. As $\hat{\sigma}_{z}$ is an even-parity operator, real level transitions are forbidden (see the Methods section). We note that the second $\hat{\sigma}_{z}$ term of $\hat{H}_{\mathrm{OP}}^{(2)}$ renormalizes the qubit transition frequency slightly and can be neglected in equation (1), which describes the real level transitions corresponding to our spectroscopy peaks. The intimate nature of the symmetry breaking resides in the coexistence of $\hat{\sigma}_{x}$ and $\hat{\sigma}_{z}$ operators in the first-order hamiltonian $\hat{H}_{\mathrm{d}}$, which produces a non-vanishing $\hat{\sigma}_{x}$ term in the second-order hamiltonian $\hat{H}^{(2)}$ of equation (1). This scenario can also be realized at the qubit optimal point by the fluctuator terms $\hat{\sigma}_{x}^{\star}$ and $\hat{\sigma}_{z}^{\star}$. As shown in Fig. 4, their presence causes a revival of the two-photon signal and the discussed strict selection rules no longer apply. Accordingly, we observe only a reduction instead of a complete suppression of the two-photon peaks near the qubit optimal point in the experimental data of Fig. $2 b$.

In summary, we use two-photon qubit spectroscopy to study the interaction of a superconducting flux qubit with an $L C$ resonator. We show experimental evidence for the presence of an anticrossing under two-photon driving, enabling us to estimate the vacuum Rabi coupling. Our experiments and theoretical analysis shed new light on the fundamental symmetry properties of quantum circuits and the nonlinear dynamics inherent to circuit QED. This can be exploited in a wide range of applications such as 
parametric upconversion, generation of microwave single photons on demand ${ }^{11,26,27}$ or squeezing ${ }^{28}$.

\section{METHODS}

\section{TWO-PHOTON-DRIVEN JAYNES-CUMMINGS MODEL VIA DYSON SERIES}

We now derive the effective second-order hamiltonian describing the physics relevant for the analysis of the two-photon-driven system. We start from the first-order hamiltonian in the basis $| \pm\rangle$,

$$
\hat{H}=\frac{\epsilon}{2} \hat{\sigma}_{z}+\frac{\Delta}{2} \hat{\sigma}_{x}+\hbar \omega_{\mathrm{r}}\left(\hat{a}^{\dagger} \hat{a}+\frac{1}{2}\right)+\hbar g \hat{\sigma}_{z}\left(\hat{a}^{\dagger}+\hat{a}\right)+\frac{\Omega}{2} \hat{\sigma}_{z} \cos \omega t .
$$

Here, in comparison with $\hat{H}_{\mathrm{d}}$, the terms associated with the fluctuator are not included $\left(\epsilon^{\star}=\Delta^{\star}=\Omega^{\star}=0\right)$ because the important features are contained in the driven qubit-resonator system. In addition, we focus on the two-photon resonance condition $\omega_{\mathrm{r}}=\omega_{\mathrm{q}}=2 \omega$. Thus, the driving angular frequency $\omega$ is largely detuned from $\omega_{\mathrm{r}}$ and the corresponding term in $\hat{H}_{\mathrm{d}}$ can be neglected $(\eta=0)$. Next, we transform the qubit into its energy eigenframe and move to the interaction picture with respect to qubit and resonator, $\hat{\sigma}_{ \pm} \rightarrow \hat{\sigma}_{ \pm} \mathrm{e}^{ \pm i \omega_{\mathrm{q}} t}, \hat{a} \rightarrow \hat{a} \mathrm{e}^{-i \omega_{\mathrm{r}} t}$ and $\hat{a}^{\dagger} \rightarrow \hat{a}^{\dagger} \mathrm{e}^{+i \omega_{\mathrm{r}} t}$. After a rotating-wave approximation, we identify the expression $\hat{S}^{\dagger} \mathrm{e}^{+i \omega t}+\hat{S} \mathrm{e}^{-i \omega t}$, where the superoperator $\hat{S} \equiv(\Omega / 4)\left(\cos \theta \hat{\sigma}_{z}-\sin \theta \hat{\sigma}_{-}\right)$and its hermitian conjugate $\hat{S}^{\dagger} \equiv(\Omega / 4)\left(\cos \theta \hat{\sigma}_{z}-\sin \theta \hat{\sigma}_{+}\right)$. In our experiments, the twophoton driving of the qubit is weak, that is, the large-detuning condition $\omega_{\mathrm{q}}-\omega=\omega \gg(\Omega / 2) \sin \theta$ is fulfilled. In such a situation, it can be shown that the Dyson series for the evolution operator associated with the time-dependent hamiltonian $-\hbar g \sin \theta\left(\hat{\sigma}_{+} \hat{a}+\hat{\sigma}_{-} \hat{a}^{\dagger}\right)+\left(\hat{S} \mathrm{e}^{-i \omega t}+\hat{S}^{\dagger} \mathrm{e}^{+i \omega t}\right)$ can be rewritten in an exponential form $\hat{U}=\mathrm{e}^{-i \hat{H}_{\text {eff }} t / \hbar}$, where

$$
\begin{aligned}
\hat{H}_{\text {eff }}= & -\hbar g \sin \theta\left(\hat{\sigma}_{+} \hat{a} \mathrm{e}^{+i \delta t}+\hat{\sigma}_{-} \hat{a}^{\dagger} \mathrm{e}^{-i \delta t}\right)+\frac{\left[\hat{S}^{\dagger}, \hat{S}\right]}{\hbar \omega} \\
= & -\hbar g \sin \theta\left(\hat{\sigma}_{+} \hat{a} \mathrm{e}^{+i \delta t}+\hat{\sigma}_{-} \hat{a}^{\dagger} \mathrm{e}^{-i \delta t}\right) \\
& +\frac{\Omega^{2}}{4 \Delta}\left(\sin ^{2} \theta \cos \theta \hat{\sigma}_{x}+\frac{1}{2} \sin ^{3} \theta \hat{\sigma}_{z}\right) .
\end{aligned}
$$

Here, $\delta \equiv \omega_{\mathrm{q}}-\omega_{\mathrm{r}}$ is the qubit-resonator detuning. In equation (2), the dispersive shift $\left(\Omega^{2} / 8 \Delta\right) \sin ^{3} \theta \hat{\sigma}_{z}$ is a reminiscence of the full second-order $\hat{\sigma}_{z}$ component of the interaction hamiltonian, $\left(\Omega^{2} / 16 \Delta\right) \sin ^{3} \theta \hat{\sigma}_{z}\left(\mathrm{e}^{+i \omega t}+\mathrm{e}^{-i \omega t}\right)^{2}$. The terms proportional to $\hat{\sigma}_{z} \exp ^{ \pm i 2 \omega t}$ are neglected implicitly by a rotating-wave approximation when deriving the effective hamiltonian $\hat{H}_{\text {eff }}$ of equation (2). In this equation, the $\hat{\sigma}_{z}$ term renormalizes the qubit transition frequency, and, in the vicinity of the anticrossing $\left(|\delta| \lesssim g \sin \tilde{\theta}, \sin \tilde{\theta}=\left(\Delta / \omega_{\mathrm{r}}\right) \simeq 0.63\right)$, the hamiltonian $\hat{H}^{(2)}$ of equation (1) can be considered equivalent to $\hat{H}_{\text {eff }}$. In this situation, the symmetries of the system are broken and our experiments demonstrate the existence of real level transitions.

\section{SELECTION RULES}

The potential of the three-Josephson-junction flux qubit can be reduced to a one-dimensional double well with respect to the phase variable $\hat{\varphi}_{\mathrm{m}}$ (ref 20). At the optimal point $\left(\Phi_{\mathrm{x}}=1.5 \Phi_{0}\right)$, this potential is a symmetric function of $\hat{\varphi}_{\mathrm{m}}$. For our experimental parameters, we can assume an effective two-level system. The two lowest energy eigenstates $|\mathrm{g}\rangle$ and $|\mathrm{e}\rangle$ are, respectively, symmetric and antisymmetric superpositions of $|+\rangle$ and $|-\rangle$. Thus, $|\mathrm{g}\rangle$ has even parity and $|\mathrm{e}\rangle$ is odd. In this situation, the parity operator $\hat{\Pi}$ can be defined via the relations $\hat{\Pi}|\mathrm{g}\rangle=+|\mathrm{g}\rangle$ and $\hat{\Pi}|\mathrm{e}\rangle=-|\mathrm{e}\rangle$. The hamiltonian of the classically driven qubit is $(\Delta / 2) \hat{\sigma}_{z}+(\Omega / 2) \cos \omega t \hat{\sigma}_{x}$. For a one-photon driving, $\omega=\Delta / \hbar$ (energy conservation), the hamiltonian in the interaction picture is $(\Omega / 4) \hat{\sigma}_{x}$, where $\hat{\sigma}_{x} \equiv|\mathrm{g}\rangle\langle\mathrm{e}|+| \mathrm{e}\rangle\langle\mathrm{g}|$. This is an odd-parity operator because the anticommutator $\left\{\hat{\Pi}, \hat{\sigma}_{x}\right\}=0$ and, consequently, one-photon transitions are allowed. For a two-photon driving, $\omega=\Delta / 2 \hbar$ (energy conservation), the effective interaction hamiltonian becomes $\left(\Omega^{2} / 8 \Delta\right) \hat{\sigma}_{z}$, where $\hat{\sigma}_{z} \equiv|\mathrm{e}\rangle\langle\mathrm{e}|-| \mathrm{g}\rangle\langle\mathrm{g}|$. As the commutator $\left[\hat{\Pi}, \hat{\sigma}_{z}\right]=0$, this is an even-parity operator and two-photon transitions are forbidden ${ }^{29}$. These selection rules are analogous to those governing electric dipole transitions in quantum optics. On the contrary, in circuit QED the qubit can be biased away from some optimal point. In this case, the symmetry is broken in a controlled way and the discussed selection rules do not hold. Instead, we find the finite transition matrix elements $(\Omega / 4) \sin \theta$ and $\left(\Omega^{2} / 4 \Delta\right) \sin ^{2} \theta \cos \theta$ for the one- and two-photon process respectively. Beyond the two-level approximation, the selection rules for a flux qubit at the optimal point are best understood by the observation that the double-well potential is symmetric there (Fig. 1d). Hence, the interaction operator of the one-photon driving is odd with respect to the phase variable $\hat{\varphi}_{\mathrm{m}}$ of the qubit potential ${ }^{20,21}$, whereas the one of the two-photon driving is even. Away from the optimal point $\left(\Phi_{\mathrm{x}} \neq 1.5 \Phi_{0}\right)$, the qubit potential has no well-defined symmetry and no selection rules apply.

\section{SPURIOUS FLUCTUATORS}

The presence of spurious fluctuators in qubits based on Josephson junctions has already been reported previously ${ }^{23}$. In principle, such fluctuators can be either resonators or two-level systems. As our experimental data does not enable us to distinguish between these two cases, for simplicity, we assume a two-level system in the simulations. In the numerical fit shown in Fig. 2a, we choose $\epsilon^{\star}=0$ owing to the limited experimental resolution. Consequently, the coupling constant estimated from the undriven fit is not $g^{\star}$, but $g^{\star} \sin \theta^{\star}$. Away from the qubit optimal point, especially near the qubit-resonator anticrossings, the effect of the observed fluctuator can be neglected within the scope of this study. Near the optimal point, its effect on the symmetry properties of the system can be explained following similar arguments as given above for the flux qubit. However, it is important to note that, differently from $\sin \theta$ and $\cos \theta$, the fluctuator parameters $\sin \theta^{\star}$ and $\cos \theta^{\star}$ are constants, that is, they do not depend on the quasi-static flux bias $\Phi_{\mathrm{x}}$.

\section{HARMONICITY OF THE LC RESONATOR}

We now show that our $L C$ resonator is harmonic and is not populated directly by two-photon driving. Anharmonicities only arise for strong driving, when the maximum induced current density $J_{\max }$ in the $L C$ resonator approaches the critical current density of the aluminium lines ${ }^{30}, J_{\mathrm{c}} \simeq 10^{7} \mathrm{~A} \mathrm{~cm}^{-2}$. From qubit Rabi oscillation measurements (data not shown), we determine the antenna current $I_{\mathrm{a}} \lesssim 1 \mu$ A resulting in a maximum resonator current $I_{\max }=M_{\mathrm{ar}} I_{\mathrm{a}} / L_{\mathrm{r}} \simeq 25 \mathrm{nA}$. Here, $L_{\mathrm{r}} \simeq 200 \mathrm{pH}$ is the resonator self-inductance and $M_{\mathrm{ar}} \simeq 5 \mathrm{pH}$ is the resonator-antenna mutual inductance. Assuming that the supercurrent flows only within the London penetration depth $\lambda_{\mathrm{L}}=50 \mathrm{~nm}$, we obtain $J_{\max } \simeq 2.5 \times 10^{2} \mathrm{~A} \mathrm{~cm}^{-2}$ for our 100-nm-thick film. As $J_{\max } / J_{\mathrm{c}}<10^{-4}$, anharmonicities can be neglected safely. Indeed, in the spectroscopy data of Fig. 2b, we observe a pronounced flux-independent one-photon excitation signal of the resonator. On the contrary, two-photon excitation peaks exclusively occur when the qubit is two-photon driven. In other words, the data unambiguously shows that there is no direct two-photon excitation of our resonator.

Received 7 February 2008; accepted 28 May 2008; published 29 June 2008.

\section{References}

1. Makhlin, Y., Schön, G. \& Shnirman, A. Quantum-state engineering with Josephson-junction devices. Rev. Mod. Phys. 73, 357-400 (2001).

2. Wendin, G. \& Shumeiko, V. S. in Handbook of Theoretical and Computational Nanotechnology Vol. 3 (eds Rieth, M. \& Schommers, W.) 223-309 (American Scientific Publishers, Los Angeles, 2006).

3. Nakamura, Y., Pashkin, Yu. A. \& Tsai, J. S. Rabi oscillations in a Josephson-junction charge two-level system. Phys. Rev. Lett. 87, 246601 (2001).

4. Oliver, W. D. et al. Mach-Zehnder interferometry in a strongly driven superconducting qubit. Science 310, 1653-1657 (2005)

5. Saito, S. et al. Parametric control of a superconducting flux qubit. Phys. Rev. Lett. 96, 107001 (2006).

6. Sillanpää, M., Lehtinen, T., Paila, A., Makhlin, Y \& Hakonen, P. J. Continuous-time monitoring of Landau-Zener interference in a Cooper-pair box. Phys. Rev. Lett. 96, 187002 (2006).

7. Wilson, C. M. et al. Coherence times of dressed states of a superconducting qubit under extreme driving. Phys. Rev. Lett. 98, 257003 (2007).

8. Wallraff, A. et al. Strong coupling of a single photon to a superconducting qubit using circuit quantum electrodynamics. Nature 431, 162-167 (2004).

9. Chiorescu, I. et al. Coherent dynamics of a flux qubit coupled to a harmonic oscillator. Nature $\mathbf{4 3 1}$ 159-162 (2004)

10. Johansson, J. et al. Vacuum Rabi oscillations in a macroscopic superconducting qubit LC oscillator system. Phys. Rev. Lett. 96, 127006 (2006)

11. Houck, A. A. et al. Generating single microwave photons in a circuit. Nature 449, 328-331 (2007).

12. Astafiev, O. et al. Single artificial-atom lasing. Nature 449, 588-590 (2007).

13. Sillanpää, M. A., Park, J. I. \& Simmonds, R. W. Coherent quantum state storage and transfer between two phase qubits via a resonant cavity. Nature $449,438-442$ (2007).

14. Majer, J. et al. Coupling superconducting qubits via a cavity bus. Nature 449, 443-447 (2007).

15. Wallraff, A. et al. Sideband transitions and two-tone spectroscopy of a superconducting qubit strongly coupled to an on-chip cavity. Phys. Rev. Lett. 99, 050501 (2007).

16. Thompson, R. J., Rempe, G. \& Kimble, H. J. Observation of normal-mode splitting for an atom in an optical cavity. Phys. Rev. Lett. 68, 1132-1135 (1992).

17. Mabuchi, H. \& Doherty, A. C. Cavity quantum electrodynamics: coherence in context. Science $\mathbf{2 9 8}$ 1372-1377 (2002)

18. Haroche, S. \& Raimond, J.-M. Exploring the Quantum (Oxford Univ. Press, New York, 2006).

19. Walther, H., Varcoe, B. T. H., Englert, B.-G. \& Becker, T. Cavity quantum electrodynamics. Rep. Prog. Phys. 69, 1325-1382 (2006).

20. Orlando, T. P. et al. Superconducting persistent-current qubit. Phys. Rev. B 60, 15398-15413 (1999).

21. Liu, Y-X, You, J. Q., Wei, L. F., Sun, C. P. \& Nori, F. Optical selection rules and phase-dependent adiabatic state control in a superconducting quantum circuit. Phys. Rev. Lett. 95, 087001 (2005). 
22. Blais, A., Huang, R.-S., Wallraff, A., Girvin, S. M. \& Schoelkopf, R. J. Cavity quantum electrodynamics for superconducting electrical circuits: An architecture for quantum computation. Phys. Rev. A 69, 062320 (2004).

23. Simmonds, R. W. et al. Decoherence in Josephson phase qubits from junction resonators. Phys. Rev Lett. 93, 077003 (2004).

24. Deppe, F. et al. Phase coherent dynamics of a superconducting flux qubit with capacitive bias readout. Phys. Rev. B 76, 214503 (2007).

25. Kakuyanagi, K. et al. Dephasing of a superconducting flux qubit. Phys. Rev. Lett. 98, 047004 (2007) 26. Mariantoni, M. et al. On-chip microwave Fock states and quantum homodyne measurements. Preprint at $<$ http://arxiv.org/abs/cond-mat/0509737> (2005).

27. Liu, Y-X, Wei, L. F. \& Nori, F. Generation of non-classical photon states using a superconducting qubit in a microcavity. Europhys. Lett. 67, 941-947 (2004).

28. Moon, K. \& Girvin, S. M. Theory of microwave parametric down-conversion and squeezing using circuit QED. Phys. Rev. Lett. 95, 140504 (2005)
29. Cohen-Tannoudji, C., Diu, B. \& Laloë, F. Quantum Mechanics (Wiley-Interscience, New York, 1977). 30. Buckel, W. \& Kleiner, R. Superconductivity (Wiley-VCH, Berlin, 2004).

\section{Acknowledgements}

We thank H. Christ for fruitful discussions. This work is supported by the Deutsche Forschungsgesellschaft through the Sonderforschungsbereich 631. Financial support by the Excellence Cluster 'Nanosystems Initiative Munich (NIM)', the EuroSQIP EU project, the Ikerbasque Foundation and UPV-EHU Grant GIU07/40 is gratefully acknowledged. This work is partially supported by CREST-JST, JSPS-KAKENHI(18201018) and MEXT-KAKENHI(18001002).

Author information

Reprints and permission information is available online at http://npg.nature.com/reprintsandpermissions. Correspondence and requests for materials should be addressed to F.D. 